\title{
Avaliação da contaminação ambiental por metais pesados, através da análise de peixes coletados da Lagoa Mangueira/RS
}

A presença de metais pesados no ambiente aquático pode ser originada de forma natural, por intemperismo das rochas ou por ações antropogênicas, com lançamento de resíduos e esgoto sem tratamento. O objetivo do presente estudo foi determinar a concentração dos metais $\mathrm{Cr}, \mathrm{Cu}, \mathrm{Ni}, \mathrm{Pb}$ e $\mathrm{Zn}$ em amostras de peixes, como o jundiá (Rhamdia quelen), traíra (Hoplias malabaricus), voga (Cyphocharax voga) e joaninha (Crenicichla punctata) coletados da Lagoa Mangueira RS. Analisou-se os tecidos dos músculos, brânquias e vísceras dos peixes. Dois métodos de digestão foram avaliados na decomposição do músculo do jundiá (Rhamdia quelen) e o método escolhido digeriu as amostras de tecido dos peixes em solução de HNO3/H2O2 2:1 (v/v), sob ultrassom à 70으 por 10 min. Em paralelo, foi analisado o material certificado DORM-3 para avaliar a exatidão do método empregado na digestão das amostras. A ordem de distribuição dos metais pesados nos tecidos dos músculos, brânquias e vísceras das espécies analisadas foi $\mathrm{Zn}>\mathrm{Cu}>\mathrm{Cr}>\mathrm{Pb}$, sendo o $\mathrm{Cr}$ e Pb quantificados somente nas partes investigadas da traíra (Hoplias malabaricus) e o Ni não foi detectado em nenhuma espécie. Através do cálculo do fator de bioacumulação (FBA) avaliou-se o nível de metais no sedimento que pode se tornar biodisponível para os peixes. Os analitos foram quantificados utilizando a espectrometria de absorção atômica com chama (FAAS).

\section{Evaluation of environmental contamination by heavy metals through the analysis of fish collected from Lagoa Mangueira/RS}

\begin{abstract}
The presence of heavy metals in the aquatic environment can originate naturally, due to weathering of the rocks or by anthropogenic actions, with the release of waste and untreated sewage. The objective of the present study was to determine the concentration of $\mathrm{Cr}, \mathrm{Cu}, \mathrm{Ni}, \mathrm{Pb}$ and $\mathrm{Zn}$ metals in fish samples such as jundia (Rhamdia quelen), traíra (Hoplias malabaricus), vogue (Cyphocharax voga) and ladybirds (Crenicichla punctata) collected from Lagoa Mangueira - RS. The tissues of the muscles, gills and viscera of the fish were analyzed. Two methods of digestion were evaluated in the decomposition of the jundiá muscle (Rhamdia quelen) and the method chosen digested the fish tissue samples in 2: $1 \mathrm{v} / \mathrm{v}) \mathrm{HNO} / \mathrm{H} 2 \mathrm{O} 2$ solution under ultrasound at $70 \circ \mathrm{C}$ for 10 min. In parallel, the certified material DORM-3 was analyzed to evaluate the accuracy of the method used in the digestion of samples. The order of distribution of the heavy metals in the tissues of the muscles, gills and viscera of the analyzed species was $\mathrm{Zn}>\mathrm{Cu}>\mathrm{Cr}>\mathrm{Pb}$, with $\mathrm{Cr}$ and $\mathrm{Pb}$ being quantified only in the investigated parts of the traíra (Hoplias malabaricus) and $\mathrm{Ni}$ was not detected in no species. Through the calculation of the bioaccumulation factor (FBA) the level of metals in the sediment that could become bioavailable to the fish was evaluated. The analytes were quantified using flame atomic absorption spectrometry (FAAS).
\end{abstract}

Keywords: Bioaccumulation; Mangueira Lagoon; Heavy Metals.

Topic: Desenvolvimento, Sustentabilidade e Meio Ambiente

Reviewed anonymously in the process of blind peer.
Received: 02/12/2018

Approved: 26/01/2019
Francisco Osvaldo Peres Pereira

Instituto Federal Sul-Rio-Grandense, Brasil http://lattes.cnpq.br/3744526671789412 frangian@terra.com.br

Leandro dos Santos

Instituto Federal Sul-Rio-Grandense, Brasil http://lattes.cnpq.br/0888460193720312 leokimico@hotmail.com

Pedro José Sanches Filho id

Instituto Federal Sul-Rio-Grandense, Brasil

http://lattes.cnpq.br/9785390634457316

http://orcid.org/0000-0002-9852-8940

pjsans@ibest.com.br
Referencing this:

PEREIRA, F. O. P.; SANTOS, L.; SANCHES FILHO, P. J.. Avaliação da contaminação ambiental por metais pesados, através da análise de peixes coletados da Lagoa Mangueira/RS. Revista Ibero Americana de Ciências Ambientais, v.10, n.1, p.180-194, 2019. DOI: http://doi.org/10.6008/CBPC2179-6858.2019.001.0015 


\section{INTRODUÇÃO}

A importância do conhecimento da concentração de metais, como $\mathrm{Cr}, \mathrm{Cu}, \mathrm{Ni}, \mathrm{Pb}$ e $\mathrm{Zn}$ em matrizes de origem ambiental (água e sedimento) e biológica (peixes) faz-se necessário, uma vez que, o monitoramento da qualidade da biota aquática já é visto como uma extensão necessária para a proteção da qualidade desse ecossistema. O estudo de metais tóxicos nos principais ambientes aquáticos do Rio Grande do Sul, como a Lagoa dos Patos, Lagoa Mirim, Canal São Gonçalo (canal que liga a Lagoa Mirim à Lagoa dos Patos), Lagoa Mangueira e o Saco do Laranjal vem sendo foco de investigação de vários trabalhos nos últimos 20 anos. Pode-se destacar, a avaliação geoquímica de metais em amostras de sedimentos superficiais do estuário da Lagoa dos Patos (NIENCHESKI et al., 1994; MIRLEAN et al., 2001; MIRLEAN et al., 2003; NIENCHESKI et al., 2004; SANTOS et al., 2004a; CALDAS et al., 2013; COSTA et al., 2017), da Lagoa Mirim (SANTOS et al., 2003; SANTOS et al., 2004b), do Saco do Laranjal (BETEMPS et al., 2012) e do Canal São Gonçalo (SANCHES FILHO et al., 2017). Além disso, encontra-se estudos da determinação da concentração de metais em amostras de água da Lagoa Mangueira (SANTOS et al., 2018a), Lagoa Mirim (FRIEDRICH et al., 2006), e Lagoa dos Patos (WALLNER-KERSANACH et al., 2009; BARBOSA et al., 2012; MILANI et al., 2012; NIENCHESKI et al., 2015; COSTA et al., 2013; WINDOM et al., 2003; COSTA et al., 2013; WINDOM et al., 1999) bem como, a investigação da bioacumulação de metais em amostras de espécies de peixes do Canal São Gonçalo (LEMUS et al., 2000) e da Lagoa dos Patos (SANCHES FILHO et al., 2013; KÜTTER et al., 2009).

Relatos de determinação da concentração de metais pesados em uma espécie de camarão (Farfantepenaeus paulensis) pescado na Lagoa dos Patos (PINTO et al., 2013) e do efeito de hidrocarbonetos transportados pelo escoamento urbano em análise de sedimento do Canal São Gonçalo (SANCHES FILHO et al., 2017b) são encontrados na literatura. Estudos registram os parâmetros que caracterizam a qualidade da água (NIENCHESKI et al., 2007) e a composição, padrão de distribuição e dominância de peixes em diferentes unidades de habitat da Lagoa Mangueira (ARTIOLI et al., 2009). O rendimento corporal e composição química do filé da viola (Loricariichthys anus) (BRITTO et al., 2014), a importância da descarga das águas subterrâneas para os níveis de nutrientes dissolvidos (ANDRADE et al., 2012), a relação entre o teor de matéria orgânica e a presença de metais pesados $\mathrm{Cu}, \mathrm{Cr}$, Pb e $\mathrm{Zn}$ (RODRíGUEZ et al., 2007) [32] e $\mathrm{Cu}, \mathrm{Cr}, \mathrm{Fe}, \mathrm{Ni}, \mathrm{Pb}$ e $\mathrm{Zn}$ (GRIMMLER et al., 2018) em amostras de sedimentos, a alimentação natural do peixe-rei (Odontesthes bonariensis) (PIEDRAS et al., 2005) e a determinação da concentração de metais traço $\mathrm{Cu}, \mathrm{Cr}, \mathrm{Fe}, \mathrm{Ni}, \mathrm{Pb}$ e $\mathrm{Zn}$ (SANTOS et al., 2018b) nas águas subterrâneas e superficiais da Lagoa Mangueira são os últimos trabalhos reportados na literatura que avaliam a composição e a qualidade desse ecossistema.

No entanto, percebe-se uma carência de estudos que determinem os níveis de metais pesados em amostras de sedimentos e espécies de peixes coletados da Lagoa Mangueira, quando comparado, por exemplo, com a quantidade de estudos para essas amostras coletadas da Lagoa dos Patos. Tal fato, justifica a execução de trabalhos que avaliem o impacto ambiental desse ecossistema, considerado parte de um grande sistema de lagoas costeiras do estado do Rio Grande do Sul (Patos-Mirim-Mangueira). 
A Lagoa Mangueira, por já ter sido mar um dia, é única em uma imensidão de água doce sobre um leito repleto de conchas fósseis, povoada por peixes como jundiá (Rhamdia quelen), traíra (Hoplias malabaricus), voga (Cyphocharax voga), joaninha (Crenicichla punctata), peixe-rei (Odontesthes bonariensis) e por mamíferos como a capivara, além de répteis e aves aquáticas. Por apresentar um valor de pH muito elevado, devido à composição do solo, a água da Lagoa Mangueira é o lar de uma microalga conhecida como Spirulina platensis que é capaz de absorver grande quantidade de poluentes da atmosfera e apresentar benefícios para a saúde humana ao ser utilizada na alimentação (MORAIS et al., 2009).

No ambiente aquático, o compartimento considerado mais significativo na concentração de metais é representado pelos sedimentos, que desempenham um papel fundamental na biodisponibilidade de várias espécies químicas. Os sedimentos possuem a capacidade de reter internamente e/ou superficialmente vários elementos químicos (HOROWITZ, 2009), podendo ser usados para detectar a presença de contaminantes que não permanecem solúveis após seu lançamento em águas superficiais (SILVA, 2002). A liberação de metais dos sedimentos para os corpos d'água, pode levar a um aumento na concentração destas espécies no meio aquático, possibilitando sua assimilação pelos peixes e organismos vivos presentes no meio, inclusive o homem (VOIGT et al., 2016).

Assim, a utilização de animais como bioindicadores é uma ferramenta extremamente útil na detecção de contaminantes e avaliação de impactos ambientais decorrentes de descargas de poluentes na biota aquática. Dentre os bioindicadores, os peixes constituem um barômetro muito útil do real estado de pureza de um sistema aquático, que não pode ser considerado em condições satisfatórias, se não houver a presença e a proliferação dessas espécies (SOUZA, 2001). A capacidade de absorver e acumular alguns metais, como por exemplo, As, $\mathrm{Fe}, \mathrm{Zn}, \mathrm{Pb}, \mathrm{Cu}$ e $\mathrm{Cd}$ nos seus tecidos musculares e órgãos faz com que os peixes possam ser considerados um indicador biológico da poluição por metais pesados (HASSAN et al., 2018).

Assim, o presente trabalho possuiu como objetivo determinar os níveis de metais pesados, tais como $\mathrm{Cr}, \mathrm{Cu}, \mathrm{Ni}, \mathrm{Pb}$ e $\mathrm{Zn}$ em amostras de peixes, como o jundiá (Rhamdia quelen), traíra (Hoplias malabaricus), voga (Cyphocharax voga) e joaninha (Crenicichla punctata) coletadas da Lagoa Mangueira/RS, bem como, a distribuição desses metais nos músculos, brânquias e vísceras dos peixes, objetivando avaliar o impacto do ambiente onde foram coletados. Também intuiu-se avaliar o nível de metais no sedimento que pode se tornar biodisponível para os peixes, calculando o fator de bioacumulação (FBA), a partir do estudo realizado por (GRIMMLER et al., 2018).

\section{MATERIAIS E MÉTODOS}

\section{Caracterização da Área de Estudo}

O estado do Rio Grande do Sul é privilegiado com sua planície costeira e abundância de rios, lagos, lagunas e lagoas com grande volume de água, destacando-se os ambientes hídricos da Laguna dos Patos, Lagoa Mirim e Lagoa Mangueira. Sendo que esta último tem $123 \mathrm{~km}$ de extensão por 7,6 km de largura no seu ponto mais largo e uma profundidade oscilando entre 1,2 a 7,4 m. Com exceção da descarga do Arroio 
Pastoreio, em Santa Vitória do Palmar/RS, não há aporte de água fluvial nem comunicação superficial com o Oceano Atlântico, resultando em uma área total de $800 \mathrm{~km}^{2}$. Suas águas estão interligadas e são conjuntamente utilizadas para a pesca, recreação e irrigação de lavouras de arroz, sendo esta última, uma das principais atividades econômicas da região. Toda a água é proveniente da chuva e do lençol freático, sendo o ambiente considerado fechado, frágil e com recirculação da água passando pelas lavouras de arroz.

A Lagoa Mangueira localiza-se no extremo sul da planície costeira, entre as dunas que separam o

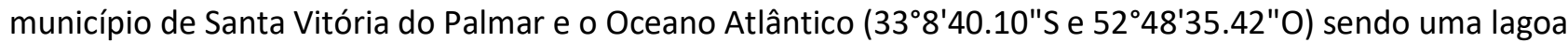
costeira bastante jovem (cerca de 4,5 mil anos), e parte integrante da Bacia da Lagoa Mirim (TOMAZELLI et al., 2005), como pode ser visto na figura 1.

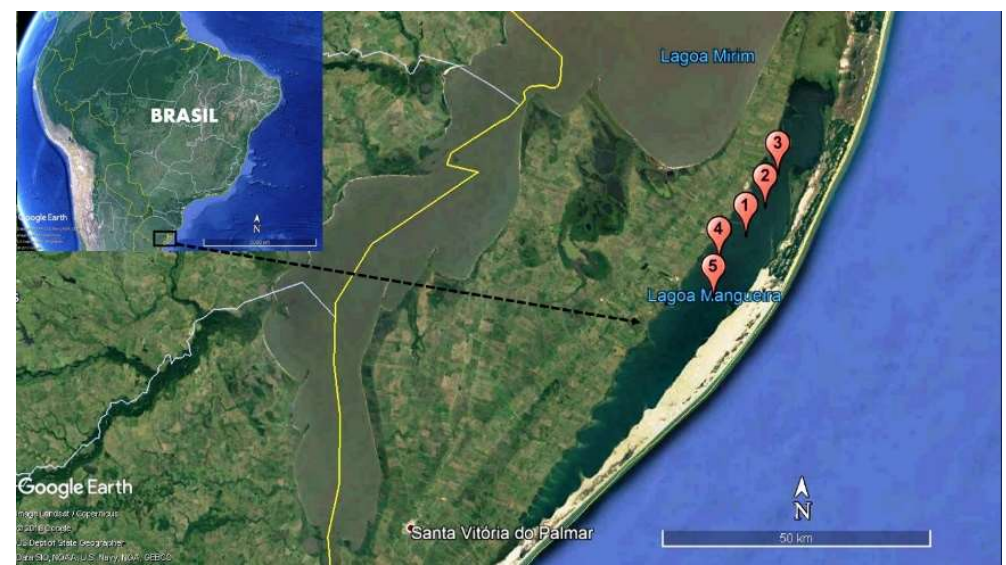

Figura 1: Localização da Lagoa Mangueira, no estado do Rio Grande do Sul - Brasil e das regiões de coleta das amostras.

Ao norte da Lagoa Mangueira está localizada a Estação Ecológica do Taim (ESECTaim), mundialmente conhecida por sua função como ponto de descanso e nidificação de aves migratórias. O maior consumo de água da Lagoa Mangueira ocorre no período de verão, devido ao cultivo de arroz na área de entorno, onde a retirada de água aliada às perdas por evaporação diminuem drasticamente os níveis de água da Lagoa (TASSI, 2008).

\section{Soluções e Reagentes}

O preparo de todas as soluções padrão, reagentes e diluições, ocorreu utilizando água purificada, com um sistema Direct-Q (3 UV, Merck Millipore, Alemanha) a uma resistividade mínima de 18,2 M $2 . s$ a 25 으. Para a quantificação dos metais, curvas de calibração para as amostras de peixes $\left(0,2-6,0 \mathrm{mg} \mathrm{L}^{-1}\right)$ foram construídas, a partir da diluição das soluções padrão de $1000 \mathrm{mg} \mathrm{L}^{-1} \mathrm{de} \mathrm{Cr}, \mathrm{Cu}, \mathrm{Ni}, \mathrm{Pb}$ e Zn (Titrisol ${ }^{\circledR}$, Merck, Alemanha), seguindo o mesmo tratamento para o material de referência certificada DORM-3 (National Research Council, Canadá).

Para o preparo das amostras utilizou-se ácido nítrico (Vetec, 65\%, Brasil), ácido clorídrico (Vetec, 3537\%, Brasil) peróxido de hidrogênio e o ácido perclórico (Labsynth, 70\%, Brasil). Todos os materiais de laboratório, como vidros, frascos de polietileno e outros recipientes foram descontaminados antes de serem utilizados, em banho de ácido nítrico a 10\% (v/v) por 24 h (WU et al., 1995). 


\section{Coleta das Amostras}

As campanhas de coleta foram realizadas no período de outubro a dezembro de 2016 , sendo coletadas amostras de peixes e sedimentos (tabela 1). 0 estudo referente aos metais presentes nos sedimentos está descrito no trabalho de Grimmler et al. (2018). Neste trabalho, serão apresentados os dados referentes à análise dos peixes, coletados utilizando redes e linhas de pesca em nylon. Foram coletadas três amostras de jundiá (Rhamdia quelen) e cinco amostras de voga (Cyphocharax voga), joaninha (Crenicichla punctata) e traíra (Hoplias malabaricus). Após a coleta, os peixes foram congelados a -4ํㅡ e transportados ao laboratório, onde foram medidos da cabeça a nadadeira caudal, antes da retirada dos músculos, brânquias e vísceras para análise dos metais e teor de umidade.

Tabela 1: Coordenadas geográficas e características dos pontos de coleta das amostras.

\begin{tabular}{|c|c|c|}
\hline Ponto & Coordenada & Especificação da área \\
\hline P1 & (331'49"S e 5242'23" O) & Região onde deságua um canal que drena a água de irrigação, lixiviada a partir das lavouras de arroz \\
\hline P2 & (3258'53"S e 5240'9" O) & Região norte da lagoa \\
\hline P3 & (3255'34"S e 5238'42" O) & \\
\hline P4 & (334'18"S e 5245'33" O) & Região central da lagoa \\
\hline P5 & (338'3"S e 5246'8" 0) & \\
\hline
\end{tabular}

\section{Preparo das Amostras}

Ao descongelarem em temperatura ambiente, procedeu-se a retirada dos tecidos dos músculos, brânquias e vísceras, que foram secos a 60ำ em uma estufa com circulação e renovação de ar (Tecnal, modelo TE-394/2, Brasil), até peso constante. A umidade das amostras, em porcentagem, foi determinada por aquecimento direto das mesmas, em forno estufa a 105으, até peso constante, de acordo o Instituto Adolfo Lutz (2008).

A etapa de digestão da amostra foi desenvolvida, avaliando a eficiência do método 1 (M1) (DURAL et al., 2007) e do método 2 (M2) (KAZI et al., 2009) em decompor o músculo do jundiá (Rhamdia quelen) e o material de referência DORM-3, com a utilização de um banho de ultrassom (Unique, USC - 4800A, Brasil), como pode ser visualizado na figura 2.

Um espectrômetro (PerkinElmer, AAnalyst 200), equipado com lâmpada de deutério como corretor de fundo e lâmpadas de catodo-oco monoelementares, foi utilizado para a obtenção das medidas analíticas, realizando a quantificação dos metais com o emprego da espectrometria de absorção atômica com chama (FAAS). A mistura ar-acetileno foi utilizada na etapa de atomização dos analitos, quantificados no comprimento de onda de maior sensibilidade analítica. O limite de quantificação (LOQ) e o limite de deteç̧ão (LOD) foram calculados considerando-se a média do sinal do branco ( $\mathrm{n}=10$ leituras) somada 10 vezes a medida do desvio padrão, para o cálculo do LOQ e 3 vezes a medida do desvio padrão, para o cálculo do LOD, conforme IUPAC (1997). 


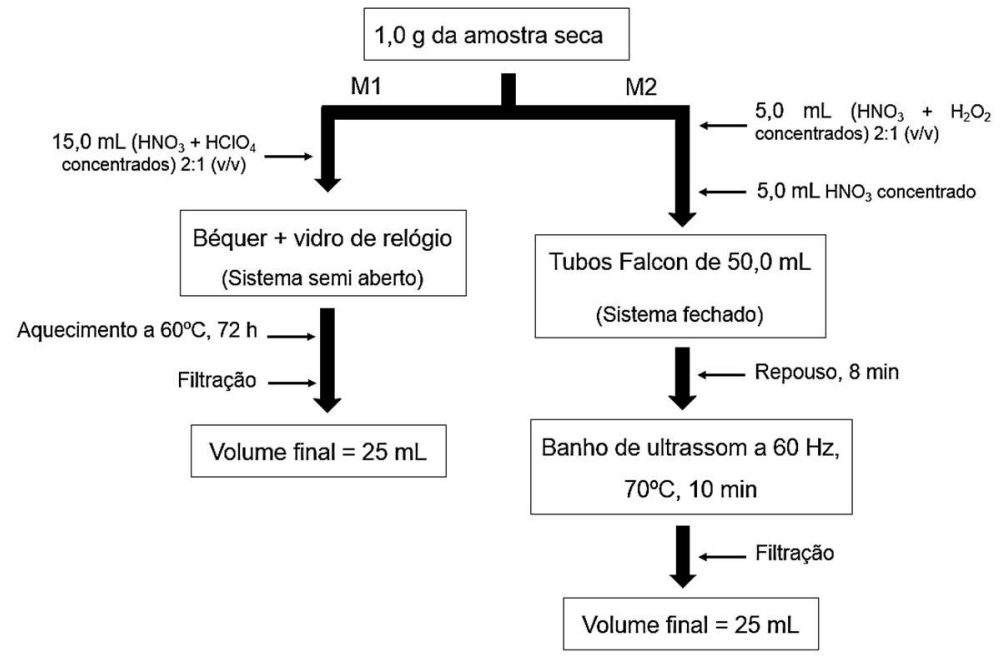

Figura 2: Procedimento desenvolvido para avaliar a eficiência dos métodos $1(\mathrm{M} 1)$ e $2(\mathrm{M} 2)$ na digestão do músculo do jundiá (Rhamdia quelen).

\section{RESULTADOS E DISCUSSÃO}

\section{Caracterização preliminar}

Trabalhos realizados por Oliveira et al. (2014), Banze (2015), Decarli et al. (2016), Lanzarin et al. (2017) e Mesquita et al. (2018) avaliaram o teor de umidade em diferentes espécies de peixes e encontraram a variação do teor na faixa de 72,7 a $78,0 \%$, que está de acordo com a faixa de 65,4 a $78,9 \%$, obtida nesse trabalho (tabela 2). Os valores encontrados na medida do comprimento e na determinação do teor de umidade indicam não haver homogeneidade entre as amostras. Esse resultado é esperado e aceitável, uma vez que os dados analíticos variam grandemente, de acordo com a espécie, idade, dieta, estação do ano que a coleta foi realizada e com o sexo dos peixes, onde os machos e fêmeas foram analisados indistintamente. Contudo, aplicando o teste $t$ (de Student) $\operatorname{com} \alpha=0,05$, não foram observadas diferenças significativas entres os resultados do teor de umidade dos músculos, vísceras e brânquias entre as espécies.

Tabela 2: Caracterização preliminar dos peixes coletados na Lagoa Magueira/RS.

\begin{tabular}{|l|l|l|l|l|}
\hline Espécie & Comprimento $(\mathrm{cm})$ & \multicolumn{3}{l|}{ Umidade $(\%) \pm \mathrm{RSD}^{(3)}$} \\
\cline { 3 - 5 } & & Músculos & Vísceras & Brânquias \\
\hline Voga (Cyphocharax voga) & $23,8 \pm 11,7^{(1)}$ & $76,4 \pm 0,7$ & $74,6 \pm 2,5$ & $66,5 \pm 5,4$ \\
\hline Traíra (Hoplias malabaricus) & $31,2 \pm 11,6^{(1)}$ & $76,3 \pm 1,6$ & $78,2 \pm 1,4$ & $69,9 \pm 1,3$ \\
\hline Joaninha (Crenicichla punctata) & $33,2 \pm 11,9^{(1)}$ & $75,3 \pm 3,1$ & $78,7 \pm 9,8$ & $65,4 \pm 3,2$ \\
\hline Jundiá (Rhamdia quelen) & $41,0 \pm 2,4^{(2)}$ & $78,9 \pm 0,7$ & $78,3 \pm 7,4$ & $71,8 \pm 2,9$ \\
\hline
\end{tabular}

${ }^{(1)} \mathrm{n}=5 ;{ }^{(2)} \mathrm{n}=3$; ${ }^{(3)}$ Desvio padrão relativo (\%).

\section{Seleção do método}

A tabela 3 apresenta os resultados obtidos no emprego dos métodos 1 e 2 na determinação dos metais $\mathrm{Zn}, \mathrm{Cr}, \mathrm{Cu}, \mathrm{Pb}$ e Ni no músculo do jundiá (Rhamdia quelen) em base seca. Ao comparar os métodos 1 e 2 percebe-se que, ambos os métodos, quantificaram os metais $\mathrm{Zn}, \mathrm{Cr}$ e Cu no material de referência, e o $\mathrm{Zn}$ e $\mathrm{Cu}$ nas amostras analisadas, obtendo valores de recuperação e desvio padrão relativo (RSD) considerados satisfatórios, de acordo com as diretrizes estabelecidas pela AOAC (2012). O método 2 apresentou maior precisão (RSD variando de 0,2 a 2,9\%), em relação ao método 1 (RSD variando de 0,5 a 9,3\%), bem como, menor tempo de execução e o uso de reagentes menos agressivos, sendo o método 
escolhido para ser empregado na determinação dos metais nos músculos, brânquias e vísceras dos peixes estudados. Em função do teor de $\mathrm{Pb}$ se encontrar abaixo do LOD e o Ni abaixo do LOQ, esses analitos não foram quantificados, tanto no material de referência, como na amostra analisada. Não foram observadas, com a aplicação do teste $t$ de Student $(\alpha=0,05)$, diferenças significativas entre os níveis de analitos quantificados pelos dois métodos.

Tabela 3: Resultados obtidos pelos métodos 1 e 2 na determinação dos metais no músculo de jundiá (Rhamdia quelen).

\begin{tabular}{|l|l|l|l|l|l|l|l|}
\hline & \multicolumn{9}{|c|}{ Método 1} & \multicolumn{2}{l|}{ Método 2 } \\
\hline Metal & $\mathrm{VC}^{(1)}$ & $\mathrm{VD}^{(2)}$ & $\operatorname{Rec}^{(3)}$ & Amostra & VD & Rec & Amostra \\
\hline $\mathrm{Zn}$ & $51,3 \pm 3,1$ & $49,9 \pm 9,3 \%$ & 97,3 & $6,8 \pm 0,5 \%$ & $55,2 \pm 0,9 \%$ & 107,5 & $6,3 \pm 0,2 \%$ \\
\hline $\mathrm{Cr}$ & $1,89 \pm 0,17$ & $1,8 \pm 5,3 \%$ & 98,8 & $<\mathrm{LOD}$ & $1,7 \pm 1,2 \%$ & 91,0 & $<$ LOD \\
\hline $\mathrm{Cu}$ & $15,50 \pm 0,63$ & $14,40 \pm 5,0 \%$ & 93,2 & $4,5 \pm 2,2 \%$ & $13,3 \pm 2,2 \%$ & 85,7 & $4,6 \pm 2,9 \%$ \\
\hline $\mathrm{Pb}$ & $0,395 \pm 0,050$ & $<\mathrm{LOD}$ & - & $<\mathrm{LOD}$ & $<\mathrm{LOD}$ & - & $<$ LOD \\
\hline $\mathrm{Ni}$ & $1,28 \pm 0,24$ & $<\mathrm{LOQ}$ & - & $<\mathrm{LOD}$ & $<$ LOQ & - & $<$ LOD \\
\hline
\end{tabular}

${ }^{(1)}$ Valor certificado do material de referência DORM-3, $\mathrm{mg} \mathrm{Kg}^{-1} \pm$ SD; (2)Valor Determinado e Amostra expressos em média $\pm \mathrm{RSD}, \mathrm{mg} \mathrm{Kg}^{-1} \pm \mathrm{RSD}(\%)$; ${ }^{(3)}$ Recuperação, (\%).

\section{Parâmetros de mérito}

A tabela 4 apresenta os parâmetros de mérito do método empregado na determinação dos metais $\mathrm{Zn}, \mathrm{Cr}, \mathrm{Cu}, \mathrm{Pb}$ e Ni nas amostras de peixes coletados na Lagoa Mangueira - RS. Observando os valores do coeficiente angular, percebe-se que o método empregado apresentou sensibilidade adequada na quantificação dos analitos estudados, principalmente, para o $\mathrm{Pb}, \mathrm{Ni}$ e $\mathrm{Cr}$.

As curvas de calibração apresentaram linearidade adequada, na faixa de concentração estudada, com coeficientes de correlação linear $(r)>0,99$ para todos os analitos estudados e RSD $(1,3$ a 2,1\%), considerados satisfatórios, de acordo com os parâmetros estabelecidos pelo mapa de 2009. A precisão das medidas foi avaliada pela injeção de 11 vezes do padrão de $2,0 \mathrm{mg} \mathrm{L}^{-1}$, de cada analito e os limites de detecção e quantificação obtidos foram adequados à proposta do estudo.

Tabela 4: Parâmetros de mérito do método empregado na determinação dos metais nas amostras de peixes coletados na Lagoa Mangueira/RS.

\begin{tabular}{|c|c|c|c|c|c|c|}
\hline \multicolumn{7}{|l|}{ Peixes } \\
\hline \multirow[t]{2}{*}{ Metal } & \multirow[t]{2}{*}{$a^{(1)}$} & \multirow[t]{2}{*}{$b^{(2)}$} & \multirow[t]{2}{*}{$R^{2(3)}$} & \multirow[t]{2}{*}{ RSD (\%) } & LOD & LOQ \\
\hline & & & & & \multicolumn{2}{|c|}{$\left(\mathrm{mg} \mathrm{Kg}^{-1}\right)$} \\
\hline $\mathrm{Zn}$ & 5,10 & $-0,146$ & 0,995 & 1,8 & 0,1 & 0,4 \\
\hline $\mathrm{Cr}$ & 30,74 & $-0,319$ & 0,998 & 1,7 & 0,4 & 1,3 \\
\hline $\mathrm{Cu}$ & 8,32 & $-0,013$ & 0,994 & 1,5 & 0,3 & 1,0 \\
\hline $\mathrm{Pb}$ & 76,78 & 0,143 & 0,999 & 1,3 & 0,7 & 1,6 \\
\hline $\mathrm{Ni}$ & 33,42 & $-0,621$ & 0,996 & 2,1 & 0,4 & 1,3 \\
\hline
\end{tabular}

${ }^{(1)}$ Coeficiente angular; ${ }^{(2)}$ Coeficiente linear; ${ }^{(3)}$ Coeficiente de determinação.

\section{Determinação dos metais}

A tabela 5 apresenta a concentração, em base úmida, dos metais $\mathrm{Zn}, \mathrm{Cu}, \mathrm{Cr}$ e $\mathrm{Pb}$ nos peixes coletados na Lagoa Mangueira/RS, bem como, a distribuição desses metais nos tecidos dos músculos, brânquias e vísceras das espécies analisadas. Em geral, essa distribuição segue a seguinte ordem: $\mathrm{Zn}>\mathrm{Cu}>\mathrm{Cr}>\mathrm{Pb}$. Contudo, o $\mathrm{Cr}$ e Pb foram quantificados somente nas partes investigadas da traíra (Hoplias malabaricus) e o Ni não foi detectado em nenhuma espécie, com o método empregado. 
Os valores de máximo e mínimo correspondem às determinações realizadas em triplicata de cada parte investigada das três amostras de jundiá (Rhamdia quelen) e cinco amostras de voga (Cyphocharax voga), joaninha (Crenicichla punctata) e traíra (Hoplias malabaricus). Os valores médios expressos em base seca foram corrigidos para base úmida, utilizando os teores de umidade de 76,7\%, 68,4\% e 77,5\%, determinados para os tecidos de músculos, brânquias e vísceras, respectivamente, com o bjetivo de comparar com os Limites Máximos Toleráveis (LMT) estabelecidos pela legislação brasileira (Decreto Lei $\mathrm{n}$ 은 55.781, de 26 de março de 1965).

Dos metais estudados, o $\mathrm{Zn}$ e o $\mathrm{Cu}$ foram os únicos quantificados em todas as espécies de peixes, sendo o Zn, o elemento majoritário. Esse metal apresentou-se com o mesmo padrão de distribuição em todas as espécies, comparando as mesmas partes investigadas, e somente na traíra (Hoplias malabaricus) as concentrações médias de Zn são equivalentes nas amostras de tecido das vísceras (46,9 $\pm 10,0 \%)$ e brânquias (50,3 $\pm 1,8 \%)$, sendo maior nessa última, como também foi observado por Souza et al. (2009) e Carmo et al. (2011).

Nas outras espécies, a concentração do Zn também foi maior nas amostras de tecido das brânquias. Esse fato pode estar relacionado, com o processo de absorção desse metal, que pode acontecer tanto pela dieta, quanto pelas brânquias na filtração da água durante a respiração, sendo esse último, o mais importante. O contato direto das brânquias com o ambiente aquático a torna um órgão indicador no que diz respeito à contaminação ambiental, sendo particularmente sensíveis à presença de contaminantes no meio e os primeiros órgãos a reagirem às condições desfavoráveis de um ambiente (VOIGT et al., 2016). As brânquias são órgãos multifuncionais, que participam do transporte de íons, trocas gasosas, regulação ácidobase e excreção.

Além disso, segundo Hardy et al. (1987), o excesso de Zn nos peixes pode ser excretado através da bílis, dos resíduos intestinais ou pelas brânquias, o que justifica a maior concentração desse metal nessa parte investigada. Embora a traíra (Hoplias malabaricus) tenha sido a espécie que apresentou a concentração mais alta para o Zn, este valor ficou abaixo do LMT, permitido pela ANVISA.

Analisando os valores das concentrações médias do Cu, percebeu-se uma tendência desse metal em se concentrar nas amostras de tecido das vísceras de todas as espécies estudadas, resultado também observado por Souza et al. (2009) e Carmo et al. (2011). Esperava-se que as concentrações médias do Cu fossem superior na traíra (Hoplias malabaricus) por tratar, entre as espécies coletadas, a que se encontra no nível trófico mais elevado da cadeia alimentar. No entanto, assim como o Zn, o Cu também é controlado por homeostasia (WATANABE et al, 1997), sendo precipitado e eliminado dos peixes por enzimas, através de mecanismos de controle de absorção intestinal, descrito por Viana et al. (2012) .

Além disso, as brâquias constituem órgãos dominantes na depuração de alguns metais, em especial o $\mathrm{Zn}$ e $\mathrm{Cu}$, pela atuação das bombas proteicas de membrana, como as $\mathrm{Ca}^{2+}, \mathrm{Na}^{+}$e $\mathrm{K}^{+}$ATPases e anidrases carbônicas (ALVORADO et al., 2006). Sendo assim, as menores concentrações de Cu observadas nesse estudo indicaram possível habilidade desse órgão em metabolizar tal metal. 
Tanto o Zn quanto o Cu apresentaram-se distribuídos de maneira mais homogênea entre as amostras coletadas do jundiá (Rhamdia quelen), diferente das outras espécies, como pode ser observado nos baixos valores de RSD para os níveis de metais nas diferentes partes analisadas. Tal situação pode ser justificada, pela uniformidade das amostras coletadas que apresentaram a menor variação de tamanho (tabela 2). Essa espécie, se alimenta de invertebrados que vivem no fundo do estuário, como vermes e moluscos, e esses organismos, devido à pouca mobilidade, podem normalmente acumular esses metais, sendo inclusive chamados de filtro marinho. Esse hábito alimentar, pode justificar o maior teor de Cu encontrado na espécie do jundiá (Rhamdia quelen). No entanto, as concentrações de Cu obtidas estavam muito abaixo do LMT, não sendo possível associar possíveis fatores determinantes de contaminação às espécies analisadas.

Entre as espécies estudadas, o $\mathrm{Cr}$ foi quantificado somente na traíra (Hoplias malabaricus) e as concentrações médias desse metal apresentaram-se acima do LMT, em todas as partes investigadas. A maior concentração desse metal foi determinada na amostra de tecido das brânquias, estando de acordo com os resultados obtidos por Carmo et al. (2011). Mesmo em baixas concentrações, esse metal pode ser acumulado nos organismos aquáticos por difusão passiva, principalmente na forma carcinogênica, atravessando as membranas e promovendo a oxidação no interior das células. Vários órgãos das espécies, como rins e fígados, são prejudicados, conforme descrito por Viana et al. (2012). Semelhante ao $\mathrm{Cr}$, o Pb foi quantificado somente na traíra (Hoplias malabaricus), e as concentrações médias desse metal nas partes investigadas, apresentaram-se abaixo do LMT. Os níveis de $\mathrm{Cr}$ e Pb encontrados nessa espécie podem estar relacionados ao processo de biomagnificação desses metais, como consequência da biodisponibilidade dos mesmos, e da dieta dos peixes. Além da biomagnificação, o teor desse metal distribuído no tecido dos músculos indica uma bioacumulação, como reflexo do contato direto dos peixes com o compartimento contaminado (água ou sedimento). Assim, ambos os fenômenos sugerem o uso de peixes como indicadores biológicos para avaliar a qualidade ambiental de recursos hídricos, sendo justificado o seu uso neste estudo.

Apesar da coleta das espécies estudadas ter sido realizada em um único período do ano, o estudo realizado por Alves et al. (2018) demonstrou que a sazonalidade pode produzir efeitos biológicos adversos à biota. Todavia, esses efeitos são específicos para cada espécie de peixe e metal investigado, não sendo considerado um fator determinante na caracterização do grau de contaminação dos recursos hídricos de acordo com as diferentes estações do ano.

Os teores de $\mathrm{Cu}$ e $\mathrm{Pb}$ quantificados nas espécies analisadas permaneceram abaixo dos limites máximos de tolerância, enquanto as maiores concentrações de $\mathrm{Zn} \mathrm{e} \mathrm{Cr}$ determinadas na traíra (Hoplias malabaricus) indicaram que o sistema aquático pode estar sofrendo impacto de atividades humanas. Isso porque, a maior parte da água da Lagoa Mangueira é proveniente da chuva e do lençol freático, sendo a irrigação de lavouras de arroz a principal atividade antropogênica realizada na região.

A tabela 6 apresenta a concentração dos metais $\mathrm{Zn}, \mathrm{Cu}, \mathrm{Cr}, \mathrm{Pb}$ e Ni nos sedimentos coletados na Lagoa Mangueira/RS por Grimmler et al. (2018), bem como, os limites de TEL (Threshold Effect Level) que representam a concentração abaixo da qual raramente ocorrerão efeitos biológicos adversos à biota, e os de PEL (Probable Effect Level), que indicam a concentração acima da qual os efeitos adversos são mais prováveis 
de serem observados. Os valores de TEL e PEL são referentes aos níveis de classificação dos sedimentos (Nível

I e Nível II, respectivamente) estabelecidos pelo CONAMA (2012) , em unidade de material seco.

Tabela 5: Concentração dos metais $\mathrm{Zn}, \mathrm{Cu}, \mathrm{Cr}$ e $\mathrm{Pb}$ nas partes investigadas dos peixes coletados na Lagoa Mangueira/RS.

\begin{tabular}{|c|c|c|c|c|c|c|c|}
\hline \multirow[t]{2}{*}{ Metal } & \multirow{2}{*}{$\begin{array}{l}\text { LMT } \\
\left(\mathrm{mg} \mathrm{Kg}^{-1}\right)\end{array}$} & \multirow[t]{2}{*}{ Espécie } & \multirow[t]{2}{*}{ Tecido } & \multicolumn{4}{|c|}{ Valor $\left(\mathrm{mg} \mathrm{Kg}^{-1} \pm \mathrm{RSD}\right)$} \\
\hline & & & & Máximo & Mínimo & Médio & Base úmida \\
\hline \multirow[t]{16}{*}{$\mathrm{Zn}$} & \multirow{16}{*}{50,0} & Traíra & & & & & \\
\hline & & & Músculo & $46,1 \pm 8,9 \%$ & $22,2 \pm 10,3 \%$ & $33,0 \pm 34,6 \%$ & $7,6 \pm 34,6 \%$ \\
\hline & & & Brânquias & $165,7 \pm 2,6 \%$ & $158,5 \pm 15,8 \%$ & $161,1 \pm 1,8 \%$ & $50,3 \pm 1,8 \%$ \\
\hline & & & Vísceras & $201,6 \pm 3,1 \%$ & $170,0 \pm 9,7 \%$ & $176,9 \pm 10,0 \%$ & $46,9 \pm 10,0 \%$ \\
\hline & & Jundiá & & & & & \\
\hline & & & Músculo & $48,1 \pm 10,9 \%$ & $46,3 \pm 3,3 \%$ & $47,1 \pm 2,0 \%$ & $10,9 \pm 2,0 \%$ \\
\hline & & & Brânquias & $84,6 \pm 4,6 \%$ & $80,8 \pm 5,4 \%$ & $83,2 \pm 2,6 \%$ & $26,0 \pm 2,6 \%$ \\
\hline & & & Vísceras & $68,1 \pm 19,0 \%$ & $57,5 \pm 8,7 \%$ & $62,4 \pm 8,6 \%$ & $14,5 \pm 8,6 \%$ \\
\hline & & Voga & & & & & \\
\hline & & & Músculo & $41,1 \pm 13,3 \%$ & $20,6 \pm 8,0 \%$ & $29,7 \pm 26,3 \%$ & $6,9 \pm 26,3 \%$ \\
\hline & & & Brânquias & $133,5 \pm 2,2 \%$ & $36,2 \pm 16,9 \%$ & $63,5 \pm 84,1 \%$ & $19,8 \pm 84,1 \%$ \\
\hline & & & Vísceras & $50,7 \pm 4,7 \%$ & $23,1 \pm 3,4 \%$ & $34,8 \pm 32,3 \%$ & $8,1 \pm 32,3 \%$ \\
\hline & & Joaninha & & & & & \\
\hline & & & Músculo & $31,2 \pm 7,8 \%$ & $19,2 \pm 16,4 \%$ & $25,3 \pm 21,6 \%$ & $5,8 \pm 21,6 \%$ \\
\hline & & & Brânquias & $111,4 \pm 6,3 \%$ & $77,7 \pm 1,4 \%$ & $95,9 \pm 14,7 \%$ & $30,0 \pm 14,7 \%$ \\
\hline & & & Vísceras & $79,0 \pm 13,4 \%$ & $60,3 \pm 9,0 \%$ & $73,3 \pm 11,1 \%$ & $17,0 \pm 11,1 \%$ \\
\hline \multirow[t]{16}{*}{$\mathrm{Cu}$} & \multirow[t]{16}{*}{30,0} & Traíra & & & & & \\
\hline & & & Músculo & $4,3 \pm 22,7 \%$ & $<$ LOD & $2,6 \pm 38,3 \%$ & $0,5 \pm 38,3 \%$ \\
\hline & & & Brânquias & $2,3 \pm 14,7 \%$ & $<$ LOD & $1,6 \pm 53,1 \%$ & $0,4 \pm 53,1 \%$ \\
\hline & & & Vísceras & $4,3 \pm 13,4 \%$ & $2,5 \pm 23 \%$ & $3,3 \pm 24,1 \%$ & $0,8 \pm 24,1 \%$ \\
\hline & & Jundiá & & & & & \\
\hline & & & Músculo & $3,2 \pm 3,5 \%$ & $2,1 \pm 13,2 \%$ & $2,8 \pm 21,3 \%$ & $0,6 \pm 21,3 \%$ \\
\hline & & & Brânquias & $3,8 \pm 26,7 \%$ & $3,2 \pm 18,2 \%$ & $3,5 \pm 9,0 \%$ & $1,1 \pm 9,0 \%$ \\
\hline & & & Vísceras & $16,8 \pm 5,1 \%$ & $10,9 \pm 16,3 \%$ & $13,1 \pm 24,4 \%$ & $3,0 \pm 24,4 \%$ \\
\hline & & Voga & & & & & \\
\hline & & & Músculo & $3,9 \pm 19 \%$ & $<$ LOD & $2,4 \pm 64,4 \%$ & $0,5 \pm 64,4 \%$ \\
\hline & & & Brânquias & $5,0 \pm 27,4 \%$ & $1,8 \pm 3,6 \%$ & $2,9 \pm 66,0 \%$ & $0,9 \pm 66,0 \%$ \\
\hline & & & Vísceras & $9,0 \pm 16,3 \%$ & $2,8 \pm 6,9 \%$ & $5,9 \pm 33,5 \%$ & $1,4 \pm 33,5 \%$ \\
\hline & & Joaninha & & & & & \\
\hline & & & Músculo & $2,4 \pm 6,7 \%$ & $1,2 \pm 15,0 \%$ & $2,0 \pm 26,1 \%$ & $0,5 \pm 26,1 \%$ \\
\hline & & & Brânquias & $6,9 \pm 9,0 \%$ & $<$ LOD & $3,8 \pm 62,0 \%$ & $1,2 \pm 62,0 \%$ \\
\hline & & & Vísceras & $31,4 \pm 29,0 \%$ & $9,6 \pm 25,1 \%$ & $19,6 \pm 40,3 \%$ & $4,5 \pm 40,3 \%$ \\
\hline \multirow[t]{4}{*}{$\mathrm{Cr}$} & \multirow[t]{4}{*}{0,10} & Traíra & & & & & \\
\hline & & & Músculo & $9,1 \pm 8,1 \%$ & $2,8 \pm 14,8 \%$ & $7,0 \pm 46,3 \%$ & $1,6 \pm 46,3 \%$ \\
\hline & & & Brânquias & $30,8 \pm 4,0 \%$ & $23,6 \pm 24,0 \%$ & $26,6 \pm 10,7 \%$ & $8,3 \pm 10,7 \%$ \\
\hline & & & Vísceras & $12,2 \pm 1,1 \%$ & $2,8 \pm 20,5 \%$ & $7,7 \pm 52,5 \%$ & $1,8 \pm 52,5 \%$ \\
\hline \multirow[t]{4}{*}{$\mathrm{Pb}$} & \multirow[t]{4}{*}{2,0} & Traíra & & & & & \\
\hline & & & Músculo & $4,3 \pm 7,4 \%$ & $<\mathrm{LOQ}$ & $4,1 \pm 14,5 \%$ & $0,9 \pm 14,5 \%$ \\
\hline & & & Brânquias & $2,1 \pm 10,9 \%$ & $<\mathrm{LOQ}$ & $2,4 \pm 15,9 \%$ & $0,7 \pm 15,9 \%$ \\
\hline & & & Vísceras & $4,9 \pm 13,7 \%$ & $<L O Q$ & $4,2 \pm 23,8 \%$ & $1,0 \pm 23,8 \%$ \\
\hline
\end{tabular}

Tabela 6: Concentração dos metais $\mathrm{Zn}, \mathrm{Cu}, \mathrm{Cr}$, Pb e Ni nos sedimentos coletados na Lagoa Mangueira, Rio Grande do Sul, Brasil.

\begin{tabular}{|l|l|l|l|l|l|l|l|l|}
\hline \multirow{2}{*}{ Metal } & TEL & PEL & & P1 & P2 & P3 & P4 & P5 \\
\cline { 2 - 8 } & $\left(\mathrm{mg} \mathrm{Kg}^{-1}\right)$ & & & $\left(\mathrm{mg} \mathrm{Kg}^{-1} \pm \mathrm{RSD}\right)$ \\
$\mathrm{Zn}$ & 123,0 & 315,0 & & $4,0 \pm 3,6 \%$ & $3,2 \pm 3,8 \%$ & $2,5 \pm 0,3 \%$ & $2,5 \pm 1,3 \%$ & $4,1 \pm 2,4 \%$ \\
\hline $\mathrm{Cu}$ & 35,7 & 197,0 & $2,1 \pm 10,1 \%$ & $2,1 \pm 5,5 \%$ & $2,0 \pm 8,5 \%$ & $2,4 \pm 11,2 \%$ & $2,5 \pm 4,2 \%$ \\
\hline $\mathrm{Cr}$ & 37,3 & 90,0 & & $<$ LOQ & $<$ LOQ & $<$ LOQ & $<$ LOQ & $<$ LOQ \\
\hline $\mathrm{Pb}$ & 35,0 & 91,3 & & $<$ LOD & $<$ LOD & $<$ LOQ & $<$ LOD & $<$ LOD \\
\hline $\mathrm{Ni}$ & 18,0 & 35,9 & $<$ LOD & $<$ LOD & $<$ LOD & $<$ LOD & $0,7 \pm 6,2 \%$ \\
\hline
\end{tabular}

Fonte: Grimmler et al. (2018).

A avaliação da bioacumulação do Zn e Cu nos peixes, devido a interação com os sedimentos, se deu através do cálculo do fator de bioacumulação. 0 nível desses metais no sedimento, que pode se tornar biodisponível para os peixes, foi calculado utilizando a razão entre a concentração determinada do metal em 
cada parte investigada dos peixes e a concentração pseudototal do metal determinada nos sedimentos da Lagoa Mangueira/RS. O fator de bioacumulação não foi calculado para os demais metais, visto que, não foram quantificados nos sedimentos e peixes, concomitantemente.

Os maiores fatores de bioacumulação estão relacionados com a concentração do Zn no tecido das brânquias de todas as espécies de peixes investigadas, mostrando que a bioacumulação ocorre de forma mais intensa neste órgão, devido ao contato direto com a água durante a respiração. De maneira geral, a concentração do Zn nos peixes apresenta a seguinte tendência: brânquias > vísceras > músculos. Já para o metal $\mathrm{Cu}$, os maiores fatores de bioacumulação se distribuem na seguinte ordem: vísceras > brânquias > músculos. Estes metais podem passar para os seres humanos através da cadeia alimentar e, portanto, predispor os consumidores a possíveis riscos para a saúde. Recomenda-se a monitorização periódica destes e de outros metais potencialmente tóxicos, tanto nos peixes como no ecossistema aquático, para garantir a segurança contínua da população e avaliar o estágio de contaminação ambiental.

Com base nos resultados obtidos dos FBA (tabela 7) é possível verificar que o tecido dos músculos tem menor afinidade para a bioacumulação dos metais $\mathrm{Cu}$ e $\mathrm{Zn}$, não sendo tecidos ativos, em relação à bioacumulação, quando comparados às brânquias e vísceras, estando estes resultados de acordo com os estudos realizados por Bajc et al. (2005), Shukla et al. (2007) e Nwani et al. (2010).

Tabela 7: Fatores de bioacumulação dos metais Zn e Cu nas amostras de tecido dos peixes coletados na Lagoa Mangueira/ RS, Brasil.

\begin{tabular}{|c|c|c|c|c|}
\hline \multirow[t]{2}{*}{ Metal } & \multirow[t]{2}{*}{ Espécie } & \multicolumn{3}{|c|}{ Fator de Bioacumulação } \\
\hline & & $\mathrm{M} / \mathrm{S}$ & $\mathrm{B} / \mathrm{S}$ & $\mathrm{V} / \mathrm{S}$ \\
\hline \multirow[t]{4}{*}{$\mathrm{Zn}$} & Traíra & 2,33 & 15,43 & 14,39 \\
\hline & Jundiá & 3,34 & 7,98 & 4,45 \\
\hline & Voga & 2,12 & 6,07 & 2,48 \\
\hline & Joaninha & 1,78 & 9,20 & 5,21 \\
\hline \multirow[t]{4}{*}{$\mathrm{Cu}$} & Traíra & 0,23 & 0,18 & 0,36 \\
\hline & Jundiá & 0,27 & 0,50 & 1,35 \\
\hline & Voga & 0,23 & 0,41 & 0,63 \\
\hline & Joaninha & 0,23 & 0,54 & 2,03 \\
\hline
\end{tabular}

M/S: Músculo/Sedimento; B/S: Brânquias/Sedimento; V/S: Vísceras/Sedimento.

\section{CONCLUSÕES}

O estudo desenvolvido demonstrou que as atividades humanas têm contribuído para o aumento do nível da concentração dos metais estudados na Lagoa Mangueira/RS. Comparando-se as partes investigadas das espécies traíra (Hoplias malabaricus), jundiá (Rhamdia quelen), voga (Cyphocharax voga) e joaninha (Crenicichla punctata), os metais $\mathrm{Zn}$ e $\mathrm{Cr}$ apresentaram maior concentração no tecido das brânquias, o Cu nas vísceras e o Pb nos músculos. Esta capacidade de retenção do íon metálico nesses tecidos sugere que as espécies analisadas podem ser empregadas como bioindicadores para os metais citados, fornecendo informações sobre a capacidade de bioacumulação desses metais na Lagoa Mangueira/RS.

$\mathrm{Na}$ traíra (Hoplias malabaricus) a concentração de $\mathrm{Cu}$ e $\mathrm{Pb}$ permaneceu abaixo do limite máximo de tolerância, estabelecido pela legislação brasileira, enquanto que a concentração de $\mathrm{Zn}$ e $\mathrm{Cr}$ ficou acima, indicando que o sistema aquático pode estar sofrendo impacto de atividades humanas. As demais espécies estudadas apresentaram concentrações de $\mathrm{Zn}$ e Cu que indicam um ambiente pouco impactado por esses 
metais. Entretanto, o monitoramento dos níveis de metais potencialmente tóxicos nesse ecossistema deve ser constante, uma vez que, os peixes podem bioacumular esses metais a partir de um ambiente poluído.

Com base nos resultados obtidos dos FBA foi possível verificar que as brânquias têm maior afinidade para bioacumulação de Zn e os músculos possuem menor afinidade na bioacumulação de Cu. Estes resultados constituem-se numa contribuição para o melhor entendimento dos processos que ocorrem na região, bem como, em um banco de dados para futuros monitoramentos da Lagoa Mangueira/RS, pois são escassos estudos de caracterização e avaliação da qualidade desse ecossistema.

AGRADECIMENTOS: Os autores agradecem a propesp do IFSUL e ao curso de química pela disponibilização de estrutura e financiamentos de bolsas de iniciação científica.

\section{REFERÊNCIAS}

ALVES, M. M.; MEDINA, A. L.; PINTO, A. M. T.; ANTUNES, A. C. N.; SANCHES FILHO, P. J.; RIBEIRO, A. S.; VIEIRA, M. A. Evaluation of the Concentration of $\mathrm{Cu}, \mathrm{Zn}, \mathrm{Pb}$ and $\mathrm{Cr}$ in Different Fish Species from the São Gonçalo Channel in Pelotas-RS, Brazil. Journal of the Brazilian Chemical Society, v.29, n.2, p.285-296, 2018. DOI: http://dx.doi.org/10.21577/0103-5053.20170139

ALVORADO, N. E.; QUESADA, I.; HYLLAND, K.; MARIGÓMEZ, I.; SOTO, M.. Quantitative changes in metallothionein expression in target celltypes in the gills of turbot (Scophthalmus maximus) exposed to $\mathrm{Cd}, \mathrm{Cu}, \mathrm{Zn}$ and after a depuration treatment. Aquatic Toxicology, v.77, n.1, p.6477, 2006. DOI:

http://doi.org/10.1016/j.aquatox.2005.10.017

ANDRADE, C. F. F.; NIENCHESKI, L. F. H.; ATTSISANO, K. K.; MILANI, M. R.; SANTOS, I. R.; MILANI, I. C.. Fluxos de nutrientes associados às descargas de água subterrânea para a Lagoa Mangueira (Rio Grande do Sul, Brasil). Química Nova, v.35, n.1, p.5-10, 2012. DOI:

http://dx.doi.org/10.1590/S0100-40422012000100002

AOAC. Official Methods of Analysis. Appendix F: Guidelines for Standard Method Performance Requirements. Washington, 2012.

ARTIOLI, L. G. S.; VIEIRA, J. P.; GARCIA, A. M.; BEMVENUTI, M. A.. Distribuição, dominância e estrutura de tamanhos da assembleia de peixes da lagoa Mangueira, sul do Brasil. Iheringia. Série Zoologia, v.99, n.4, p.409-418, 2009. DOI: http://dx.doi.org/10.1590/S0073-47212009000400011

BANZE, J. F.. Silagem ácida de vísceras de atum em dietas para juvenis de jundiá (Rhamdia quelen): digestibilidade e desempenho em diferentes níveis de substituição à farinha de peixe. Dissertação (Mestrado em Aquicultura) Universidade Federal de Santa Catarina, Florianópolis, 2015.

BARBOSA, F. G.; WALLNER-KERSANACH, M.; BAUMGARTEN, M. G. Z.. Metais traço nas águas portuárias do estuário da Lagoa os Patos, RS. Brazilian Journal of Aquatic Science and Technology, v.16, n.2, p.27-36, 2012. DOI: http://dx.doi.org/10.14210/bjast.v16n2.p27-36
BETEMPS, G. R.; SANCHES FILHO, P. J.. Estudo sazonal de metais pesados no sedimentodo Saco do Laranjal - PelotasRS. Journal of the Brazilian Society of Ecotoxicology, v.7, n.2, p.79-84, 2012. DOI: http://doi.org/10.5132/jbse.2012.02.012

BAJC, Z.; GACNIK, K. S.; JENCIC, V.; DOGANOC, D. Z.. The content of $\mathrm{Cu}, \mathrm{Zn}$ and $\mathrm{Mn}$ in Slovenian freshwater fish. Slovenian Veterinary Research, v.42, n.1-2, p.15-21, 2005.

BRASIL. Decreto Lei $\mathbf{n}$.55.871: Dispões sobre os Princípios Gerais para o Estabelecimento de Níveis Máximos de Contaminantes Químicos em Alimentos e seu anexo Limites máximos de tolerância para contaminantes inorgânicos. 1965.

BRITTO, A. C. P; ROCHA, C. B.; TAVARES, R. A.; FERNANDES, J. M.; PIEDRAS, S. R. N.; POUEY, J. L. O. F.. Rendimento corporal e composição química do filé da viola (Loricariichthys anus). Ciência Animal Brasileira, v.15, n.1, p.38-44, 2014. DOI: http://dx.doi.org/10.5216/cab.v15i1.21673

CALDAS, J. S.; SANCHES FILHO, P. J.. Determinação de Cu, Pb e Zn no sedimento da região do pontal da barra, Laranjal (Laguna dos Patos, Pelotas - RS, Brasil). Brazilian Journal of Aquatic Science and Technology, v.17, n.1, p.13-18, 2013. DOI: http://dx.doi.org/10.14210/bjast.v17n1.p13-18

CARMO, C. A.; ABESSA, D. M. S.; MACHADO NETO, J. G. Metais em águas, sedimentos e peixes coletados no estuário de São Vicente - SP, Brasil. O Mundo da Saúde, v.35, n.1, p.64-70, 2011.

CONAMA. Resolução n.454/2012: Estabelece as diretrizes gerais e os procedimentos referenciais para o gerenciamento do material a ser dragado em águas sob jurisdição nacional. 2012.

COSTA, L. D. F.; CASARTELLI, M. R. O.; WALLNERKERSANACH, M.. Labile copper and zinc fractions under different salinity conditions in a shipyard area in the patos lagoon estuary, South of Brazil. Química Nova, v.36, v.8, p.1089-1095, 2013. DOI: http://dx.doi.org/10.1590/S0100$\underline{40422013000800002}$ 
COSTA, L. D. F.; WALLNER-KERSANACH, M.. Assessment of the labile fractions of copper and zinc in marinas and port areas in Southern Brazil. Environmental Monitoring and Assessment, v.185, n.8, p.6767-6781, 2013. DOI: http://doi.org/10.1007/s10661-013-3063-0

COSTA, L.; MIRLEAN, N.; GARCIA, F.. Arsenic Environmental Threshold Surpass in Estuarine Sediments: Effects of Bioturbation. Bulletin of Environmental Contamination and Toxicology, v.98, n.4, p.521-524, 2017. DOI: http://doi.org/10.1007/s00128-016-2024-z

DECARLI, J. A.; BITTARELLO, A. C.; SIVIDANES, V. P.; SARY, C.; FEIDEN, A.; SIGNOR, A.; BITTENCOURT, F.. Farinha de minhoca para juvenis de jundiá Rhamdia voulezi criados em tanques-rede. Revista Agrarian, v.9, n.34, p.390-396, 2016.

DURAL, M.; GÖKSU, M. Z. L.; ÖZAK, A. A.. Investigation of heavy metal levels in economically important fish species captured from the Tuzla lagoon. Food Chemistry, v.102, n. 1 p. 415-421, 2007. DOI:

http://doi.org/10.1016/i.foodchem.2006.03.001

FRIEDRICH, A. C.; NIENCHESKI, L. F.; SANTOS, I. R.. Dissolved and particulate metals in Mirim Lagoon, Brazil-Uruguayan Border. Journal of Coastal Research, v.39, p.1036-1039, 2006. DOI: http://www.jstor.org/stable/25741737

GRIMMLER, M. U.; PEREIRA, F. O. P.; SILVEIRA, L. A.; SANTOS, L.; SANCHES FILHO, P. J.. Determinação de metais tóxicos $\mathrm{Cr}, \mathrm{Cu}, \mathrm{Ni}, \mathrm{Pb}$ e $\mathrm{Zn}$ em amostras de sedimentos da Lagoa Mangueira-RS-Brasil. Revista Ibero-Americana de Ciências Ambientais, v.9, n.7, p.236-242, 2018.

HARDY, R. W.; SULLIVAN, C. V.; KOZIOL, A. M.. Absorption, body distribution and excretion of dietary zinc by rainbow trout (Salmo gairdneri). Fish Physiology Biochemistry, v.3, n.3, p.133-143, 1987. DOI: http://dx.doi.org/10.1007/BF02180415

HASSAN, A.; MOHARRAM, S.; EL HELALY, H.. Role of Parasitic Helminths in Bioremediating Some Heavy Metal Accumulation in the Tissues of Lethrinus mahsena. Turkish Journal of Fisheries and Aquatic Sciences, v.18, n.3, p.435443, 2018. DOI: http://doi.org/10.4194/1303-2712$\underline{v 18 \quad 3 \quad 09}$

HOROWITZ, A. J.. A Primer on sediment - trace element chemistry, 2 Ed. Chelsea Mich: Lewis Publishers, 1991.

INSTITUTO ADOLFO LUTZ. Métodos Físico-Químicos para Análise de Alimentos. 4 ed. São Paulo, 2004.

IUPAC. Chemistry Compendium of Chemical Terminology. 2 ed. 1997.

KAZI, T. G;. JAMALI, M. K.; ARAIN, M. B.; AFRIDI, H. I.; JALBANI, N.; SARFRAZ, R. A.; ANSARI, R.. Evalution of na ultrasonic acid digestion procedure for total heavy metals determination in environmental and biological samples. Journal of Hazardous Materials, v.161, n.2-3, p.1391-1398, 2009. DOI: http://doi.org/10.1016/j.jhazmat.2008.04.103

KÜTTER, V. T.; MIRLEAN, N.; NAISCH, P. R. M.; KÜTER, M. T.; SILVA-FILHO, E. V.. Mercury in freshwater, estuarine, and marine fishes from Southern Brazil and its ecological implication. Environmental Monitoring and Assessment, v.159, n.35, p.35-42, 2009. DOI:

http://doi.org/10.1007/s10661-008-0610-1

LANZARIN, M.; RITTER, D. O.; FILHO, E. S. A.; MÁRSICO, T.; FREITAS, M. Q.. Composição centesimal e teste de aceitação e intenção de compra do pintado amazônico (Pseudoplatystoma fasciatum X Leiarius marmoratus) e piauçu (Leporinus macrocephalus). Revista Brasileira de Ciência Veterinária, v.24, n.3, p.162-166, 2017. DOI: http://doi.org/10.4322/rbcv.2017.031

LEMUS, M.; TOLEDO, J.; CHUNG, K. S.. Cobre, cadmio y plomo en el pez Cyprinodon dearborni, sedimentos y agua em dos lagunas de Venezuela. Revista de Biología Tropical, v.48, n.1, p.225-231, 2000.

MESQUITA, R. C. T.; GALVÃO, J. A.; SAVAYDA-SILVA, L. K.; ELOY, L. R.; GODOY, L. C.; JR STREIT, D. P.. Protocolo para produção de tambaqui baseado na densidade de estocagem e sexo. Revista Brasileira de Higiene e Sanidade Animal, v.12, n.2, p.146-155, 2018. DOI: http://dx.doi.org/10.5935/1981-2965.20180014

MILANI, M. R.; FARIAS, J. S.; NIENCHESKI, L. F. H.; PAICA, M. L.. Especiação química de arsênio inorgânico no estuário da Laguna dos Patos (RS, Brasil). Química Nova, v.35, n.7, p.1401-1406, 2012. DOI: http://dx.doi.org/10.1590/s010040422012000700021

MIRLEAN, N.; ANDRUS, V. E.; BAISCH, P.. Mercury pollution sources in sediments of Patos Lagoon Estuary, Southern Brazil. Marine Pollution Bulletin, v.46, n.3, p.331-334, 2003. DOI: http://doi.org/10.1016/S0025-326X(02)00404-6

MIRLEAN, N.; BARAJ, B.; NIENCHESKI, L. F.; BAISCH, P.; ROBINSON, D.. The effect of acidental sulphuric acid leaking on metal distributions in estuarine sedimento of Patos Lagoon. Marine Pollution Bulletin, v.42, n.11, p.1114-1117, 2001. DOI: http://doi.org/10.1016/S0025-326X(01)00099-6

MORAIS, M. G.; RADMANN, E. M.; ANDRADE, M. R.; TEIXEIRA, G. G.; BRUSCH, L. R. F.; COSTA, J. A. V. Pilot scale semicontinuous production of Spirulina biomass in southern Brazil. Aquaculture, v. 294, n. 1-2 , p. 60-64, 2009. DOI: http://dx.doi.org/10.1016/j.aquaculture.2009.05.009

NIENCHESKI, L. F. H.; BARAJ, B.; WINDOM, H. L.; FRANÇA, R. G.. Natural background assessment and its anthropogenic contamination of $\mathrm{Cd}, \mathrm{Pb}, \mathrm{Cu}, \mathrm{Cr}, \mathrm{Zn}, \mathrm{Al}$ and $\mathrm{Fe}$ in the sediments of the southern area of Patos Lagoon. Journal of Coastal Research, n.39, p.1040-1043, 2004. DOI: http://www.jstor.org/stable/25741738

NIENCHESKI, L. F. H.; WINDOM, H. L.. Chemistry of a surficial aquifer of a Large Coastal Lagoon Barrier and its relation to adjacent surface waters of Brazil. Journal of Coastal Research, v.31, n.6, p.1417-1428, 2015. DOI: http://doi.org/10.2112/JCOASTRES-D-13-00175.1

NIENCHESKI, L. F. H.; ZEPKA BAUMGARTEN, M. G.. Water Quality in Mangueira Bay: Anthropic and Natural Contamination. Journal of Coastal Research, v.47, p.56-62, 2007. DOI: http://doi.org/10.2112/1551-5036-47.sp1.56

NIENCHESKI, L. F.; WINDOM, H. L.; SMITH, R.. Distribution of particulate trace metal in Patos Lagoon estuary (Brazil). 
Marine Pollution Bulletin, v.28, n.2, p.96-102, 1994. DOI: http://doi.org/10.1016/0025-326X(94)90545-2

NWANI, C. D.; NWACHI, D. A.; OKOGWU, O. I.; UDE, E. F.; $\mathrm{ODOH}, \mathrm{G}$. E.. Heavy metals in fish species from lotic freshwater ecosystem at Afikpo, Nigeria. Journal of Environmental Biology, v.31, n.5, p.595-601, 2010.

OLIVEIRA, P. R.; DAMASCENO, J. M. B.. Propriedades químicas e rendimento da piramutaba (Brachyplastystoma vaillantii, Valenciennes, 1840). Pubvet, Londrina, v.8, n.14 2014.

PIEDRAS, S. R. N.; POUEY, J. L. O. F.. Alimentação do peixe-rei (Odontesthes bonariensis, Atherinopsidae) nas lagoas Mirim e Mangueira, Rio Grande do Sul, Brasil. Iheringia. Série Zoologia, v.95, n.2, p.117-120, 2005. DOI: http://dx.doi.org/10.1590/S0073-47212005000200001

PINTO, A. M. T. P.; HIRDES, I. M.; SANCHES FILHO, P. J.. Determinação de metais pesados nos camarões (Farfantepenaeus paulensis) consumidos na cidade de Pelotas-RS. Ecotoxicology and Environmental Contamination, v.8, n.1, p.129-134, 2013. DOI: http://doi.org/10.5132/eec.2013.01.019

RODRÍGUEZ, A.; CORRAL, M.; ORONÁ, C.; HILLMAN, G.; PAGOT, M.; SIERRA, J. P.; NIENCHESKI, F.. Sediment Quality and Resuspension Rate in Mangueira Bay, Brazil. Journal of Coastal Research, v.47, p.69-75, 2007. DOI: http://doi.org/10.2112/1551-5036-47.sp1.69

SANCHES FILHO, P. J.; BÖHM, E. M.; BÖHM, G. M. B.; MONTENEGRO, G. O.; SILVEIRA, L. A.; BETEMPS, G. R. Determination of hydrocarbons Transported by urban runoff in sediments of São Gonçalo Channel (Pelotas - RS, Brazil). Marine Pollution Bulletin, v.114, n.2, p.1088-1095, 2017 DOI: http://dx.doi.org/10.1016/i.marpolbul.2016.10.024

SANCHES FILHO, P. J.; BÖHM, E. M.; BÖHM, G. N. B.; MONTENEGRO, G. O.; SILVEIRA, L. A.; BETEMPS, G. R. Determination of hydrocarbons transported by urban runoff in sediments of São Gonçalo Channel (Pelotas - RS, Brazil). Marine Pollution Bulletin, v.114, n.2, p.1088-1095, 2017. DOI: http://dx.doi.org/10.1016/j.marpolbul.2016.10.024

SANCHES FILHO, P. J.; FONSECA, V. K.; HOLBIG, L.. Avaliação de metais em pescado da região do Pontal da Barra, Laguna dos Patos, Pelotas-RS. Ecotoxicology and Environmental Contamination, v.8, n.1, p.105-111, 2013. DOI: http://doi.org/10.5132/eec.2013.01.015

SANTOS, F. S.; RANGEL, E. M.; SANCHES FILHO, P. J. Determination of trace metals in water from Mangueira Lagoon - RS, Brazil. Water Practice \& Technology, v.13, n.3, p.612-620, 2018a. DOI: http://doi.org/10.2166/wpt.2018.077

SANTOS, F. S.; RANGEL, E. M.; SANCHES FILHO, P. J. Determination of trace metals in water from Mangueira Lagoon - RS, Brazil. Water Practice \& Technology, v.13, n.3, p.612-620, 2018b. DOI: http://doi.org/10.2166/wpt.2018.077

SANTOS, I. R.; BAISCH, P.; LIMA, G. T. N. P.. Metais pesados em sedimentos superficiais da Lagoa Mirim, fronteira
Brasil-Uruguai. Geochimica Brasiliensis, v.17, n.1, p.37-47, 2003. DOI: http://doi.org/10.21715/gb.v17i1.196

SANTOS, I. R.; BAISCH, P.; LIMA, G.; MIRLEAN, N.; GRIEP, G.; SILVA-FILHO, E. V.. Análise estatística multivariada de parâmetros geoquímicos em sedimentos do estuário da Laguna dos Patos. Geochimica Brasiliensis, v.18, n.1, p.3845, 2004a. DOI: http://doi.org/10.21715/gb.v18i1.209

SANTOS, I. R.; BAISCH, P.; LIMA, G. T. N. P.; SILVA FILHO, E, V.. Nutrients in surface sediments of Mirim lagoon, BrazilUruguay border. Acta Limnologica Brasiliensia, v.16, n.1, p.85-94, 2004b.

SHUKLA, V.; DHANKHAR, M.; PRAKASH, J.; SASTRY, K. V. Bioaccumulation of $\mathrm{Zn}, \mathrm{Cu}$ and $\mathrm{Cd}$ in Channa punctatus. Journal of Environmental Biology, v.28, n.2, p.395-397, 2007.

SILVA, M. R. C.. Estudo de sedimentos da bacia hidrográfica do Moji-Guaçu, com ênfase na determinação de metais. Dissertação (Mestrado em Ciências Química Analítica) Universidade de São Paulo, São Carlos, 2002.

SOUZA, G. R.; GARCEZ, M. A. P.; SANTOS, V. C. G.; BEDANA, D.; CAETANO, J.; DRAGUNSKI, D. C.. Quantificação de metais pesados em peixes de um pesqueiro localizado na cidade de Umuarama - PR. Arquivos de Ciências Veterinárias e Zoologia da UNIPAR, v.12, n.1, p.61-66, 2009. DOI: http://doi.org/10.25110/arqvet.v12i1.2009.2935

SOUZA, P. A. P.. Importância do uso de bioindicadores de qualidade: o caso específico das águas. In: FELICIDADE, N. et al. Uso e gestão dos recursos hídricos no Brasil. São Carlos: Rima, 2001. p.55-66.

TASSI, R.. Gerenciamento hidroambiental de terras úmidas. Tese (Doutorado em Recursos hídricos e Saneamento ambiental) - Universidade Federal do Rio Grande do Sul, Porto Alegre, 2008.

TOMAZELLI, L. J.; VILLWOCK, J. A.. Mapeamento geológico de planícies costeiras: o exemplo da costa do Rio Grande do Sul. Gravel, n.3, p.109-115, 2005.

VIANA, Z. C. V.; SILVA, E.; FERNANDES, G. B.; KORN, M. G. A. ; SANTOS, V. L. C. S.. Estudo da absorção de elementos químicos no músculo de peixes do litoral da Bahia. Revista de Ciências Médicas e Biológicas, v.11, n.1, p.27-34, 2012. DOI: http://dx.doi.org/10.9771/cmbio.v11i1.5913

VOIGT, C. L.; SILVA, C. P.; CAMPOSA, S. X.. Avaliação da Bioacumulação de Metais em Cyprinus Carpio pela Interação com Sedimento e Água de Reservatório. Química Nova, v.39, n.2, p.180-188, 2016. DOI: http://dx.doi.org/10.5935/01004042.20160014

WALLNER-KERSANACH, M.; ANDRADE, C. F. F.; ZHANG, H.; MILANI, M. R.; NIENCHESKI, L. F. H.. In situ measurement of trace metals in estuarine waters of Patos Lagoon using diffusive gradients in thin films (DGT). Journal of the Brazilian Chemical Society, v.20, n.2, p.333-340, 2009. DOI: http://dx.doi.org/10.1590/S0103-50532009000200019 WATANABE, T.; KIRON, V.; SATOH, S.. Trace minerals in fish nutrition. Aquaculture, v.151, n.1-4, p.185-207, 1997. DOI: http://doi.org/10.1016/S0044-8486(96)01503-7 
WINDOM, H. L.; NIENCHESKI, L. F.; SMITH JR, R. G..

Biogeochemistry of nutrients and trace metals in the estuarine region of the Patos Lagoon (Brazil). Estuarine, Coastal and Shelf Science, v.48, n.1, p.113-123, 1999. DOI: http://doi.org/10.1006/ecss.1998.0410

WINDOM, H.; NIENCHESKI, F.. Biogeochemical processes in a freshwater-seawater mixing zone in permeable sediments along the coast of Southern Brazil. Marine Chemistry, v.83, n.3-4, p.121-130, 2003. DOI: http://doi.org/10.1016/S03044203(03)00106-3

WU, Q.; BATLEY, E. G.. Determination of sub-nanomolar concentrations of lead in sea water by adsorptive stripping voltammetry with xylenol orange. Analytica Chimica Acta, v.309, n.1-3, p.95-101, 1995. DOI:

http://doi.org/10.1016/0003-2670(95)00

A CBPC - Companhia Brasileira de Produção Científica (CNPJ: 11.221.422/0001-03) detém os direitos materiais desta publicação. Os direitos referem-se à publicação do trabalho em qualquer parte do mundo, incluindo os direitos às renovações, expansões e disseminações da contribuição, bem como outros direitos subsidiários. Todos os trabalhos publicados eletronicamente poderão posteriormente ser publicados em coletâneas impressas sob coordenação da Sustenere Publishing, da Companhia Brasileira de Produção Científica e seus parceiros autorizados. Os (as) autores (as) preservam os direitos autorais, mas não têm permissão para a publicação da contribuição em outro meio, impresso ou digital, em português ou em tradução. 